\title{
ZONAL COMBUSTION IN TUBES ${ }^{1}$
}

\author{
BY \\ S. A. SCHAAF \\ University of California, Berkeley
}

1. Introduction. Theoretical work on combustion of gases has been chiefly concerned with two types of approximations: (i) "constant volume" or uniform state combustion, ${ }^{2}$. where it is assumed that the state of a burning gas in a chamber is the same at all parts of the chamber at any given time; (ii) "detonation front" and "deflagration front" combustion, ${ }^{3}$ where it is assumed that combustion takes place instantaneously in an infinitesimally thin front which advances with a finite velocity through the gas. We consider here a third type of approximation where it is assumed that combustion extends over a.definite zone or region, which moves with time through the gas. This model is suggested by the appearance of turbulent combustion in tubes. We confine our attention to effectively one-dimensional phenomena, in particular to combustion in a half-open tube. A basic assumption is that at any time the effective pressure, velocity, etc. of the gas in a given cross-section of the tube can be characterized by single quantities. The important physical parameters, which will depend on the degree of turbulence in the tube, the strength of the combustible mixture, etc., are the rate with which energy is released during combustion, the velocity with which the burning region progresses into the fresh gas and the duration of burning of a given layer of gas. Explicit results are obtained good to first order terms in a perturbation expansion in a parameter proportional to the average rate of energy release.

2. Formulation. Let us consider a tube of constant cross-section and length $L$, (see Fig. 1) initially filled with a combustible gas at rest and at uniform normal temperature and pressure, open at one end and closed at the other. The combustible gas is ignited at the closed end, and the flame front travels down the tube followed by a zone of burning gas. We denote by $x$ the distance from the closed end of the tube, by $t$ the time, by $a$ the initial position $(x)$ of a given layer of gas, by $p$ the pressure and by $v$ the specific volume (reciprocal of the density). We consider the equations of the system

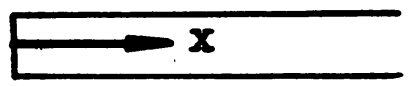

Fig. 1. Half-open tube filled with combustible gas.

in Lagrangian coordinates, so that $a$ and $t$ are the independent variables and $x=$ $x(a, t), p=p(a, t) v=v(a, t)$. Assuming that at time $t=0$ the gas in the tube is in a uniform state with $p=p_{0}, v=v_{0}$ and $x=a$, we have the equations of continuity and momentum respectively

1Received Sept. 5, 1947. This paper uses a type of formulation which was developed for the study of jet devices at the Institute of Mathematics and Mechanics of New York University by J. K. L. MacDonald and the author under a Navy contract. See $\boldsymbol{A}$ gas dynamical formulation for waves and combustion in pulse-jets, Applied Mathematics Group, New York University, Report 151. 1944.

${ }^{2} \mathrm{See}$, for example, Jennings and Obert, Internal combustion engines, International Textbook Co.,

${ }^{3}$ See, for example, R. Becker, Stosswelle und Detonation, Z. Physik, 8, 1922. 


$$
\begin{aligned}
v & =v_{0} \frac{\partial x}{\partial a} \\
\frac{\partial^{2} x}{\partial t^{2}} & =-v_{0} \frac{\partial p}{\partial a}
\end{aligned}
$$

For the energy equation, we consider a given layer of gas in which energy of amount $E$ per unit mass per unit time is being liberated by the combustion process. This energy will go partly into expansion of the layer and partly into raising the temperature." We thus have

$$
E=p \frac{\partial v}{\partial t}+\frac{\partial}{\partial t}\left(c_{v} \theta\right)
$$

as well as the gas law

$$
p v=\left(c_{p}-c_{v}\right) \theta,
$$

where $\theta$ denotes the temperature, and $c_{v}$ and $c_{p}$ are the specific heats at constant volume and pressure respectively. Eliminating the temperature we obtain the energy equation

$$
(\gamma-1) E=\gamma p \frac{\partial v}{\partial t}+v \frac{\partial p}{\partial t}
$$

where $\gamma$ is the adiabatic exponent, $\gamma=c_{p} / c_{v}$.

For the zonal type of combustion we are considering, the quantity $E$ will at any time be zero in the non-burning parts of the tube and have non-zero values depending on the time, temperature, turbulence, gas mixture, etc., in the burning part. Let $t_{0}$ be the average duration of combustion in a given layer of gas, and $\Delta E$ be the average total energy released per unit mass by the combustion; then the average value of $E$ in the burning region will be $E_{0}=\Delta E / t_{0}$. The burning region will advance into the fresh gas with a certain velocity $f$ relative to the gas, depending on the temperature, turbulence, etc. Let $f_{0}$ be the average value of $f$. Our principal idealization then is to replace the functions $f$ and $E$ by their average values, so that $f=f_{0}$, and $E=E_{0}$ in the burning region and $E=0$ in the non-burning region, ${ }^{5}$ i.e. we assume that

$$
E=\left\{\begin{array}{ccc}
E_{0} & \text { for } & f_{0} t>a>f_{0}\left(t-t_{0}\right) \quad \text { or } \quad 0 \\
0 & \text { for } & a>f_{0} t ; a<f_{0}\left(t-t_{0}\right) .
\end{array}\right.
$$

The differential system (1), (2) and (3) is then completely specified; we wish to obtain a solution of it subject to the following initial and boundary conditions,

'The complete energy balance of course includes terms accounting for heat flow and dissipation, which we consider as included in the $E$, as well as terms accounting for the kinetic energy of mass motion and work done by the pressure gradient; these latter two cancel out by virtue of the momentum equation (2), so that the energy equation as written is complete.

'It is not necessary to treat this as an idealization since these average values could be taken as only the first approximations to $E$ and $f$ in the perturbation process considered in section 3 . The results to first order terms are, of course, the same; it seems preferable for clarity and brevity of presentation to regard this form of $E$ and $f$ as idealizations. 


$$
\begin{array}{cc}
\text { at } t=0 ; \quad p=p_{0}, \quad v=v_{0}, \quad x=a \\
\frac{\partial p}{\partial t}=\frac{\partial v}{\partial t}=\frac{\partial x}{\partial t}=0 & \\
\text { at } \quad x=0: \frac{\partial x}{\partial t}=0 \quad \text { (closed end) } \\
\text { at } \quad x=L: p=p_{0} \quad \text { (open end) }
\end{array}
$$

It is convenient to introduce the dimensionless quantities

$$
\begin{aligned}
& V=v / v_{0}, \quad P=p / p_{0}, \quad X=x / L, \quad A=a / L \\
& T=c t / L, \quad T_{0}=c t_{0} / L, . \quad \lambda=f_{0} / c, \\
& \epsilon=\gamma(\gamma-1) E_{0} L / c^{3} \\
& I=\left\{\begin{array}{lll}
1 & \text { for } & \lambda T>A>\lambda\left(T-T_{0}\right), \\
0 & \text { for } & A>\lambda T \quad \text { or } \quad A<\lambda\left(T-T_{0}\right),
\end{array}\right.
\end{aligned}
$$

where $c$ is the initial sound speed, $c^{2}=\gamma p_{0} v_{0}$. The dimensionless form of (1), (2), (3) is then

$$
\begin{aligned}
V & =\frac{\partial X}{\partial A}, \\
\gamma \frac{\partial^{2} X}{\partial T^{2}} & =-\frac{\partial P}{\partial A}, \\
\gamma P \frac{\partial V}{\partial T}+V \frac{\partial P}{\partial T} & =I \epsilon .
\end{aligned}
$$

The initial and boundary conditions become

$$
\begin{gathered}
\text { at } T=0: P=V=1, \quad X=A, \\
\frac{\partial P}{\partial T}=\frac{\partial V}{\partial T}=\frac{\partial X}{\partial T}=0 \\
\text { at } X=0: \frac{\partial X}{\partial T}=0, \\
\text { at } X=1: P=1 .
\end{gathered}
$$

3. Perturbation solution. The non-linear system $\left(1^{\prime}\right),\left(2^{\prime}\right),\left(3^{\prime}\right)$ is not easily solvable in exact form, but approximate solutions can be obtained valid for "small" values of 
the parameter $\epsilon$, if we assume that a power series development of $P, V$ and $X$ in terms of $\epsilon$ is possible, so that

$$
\begin{aligned}
& P=1+\epsilon P^{\prime}(A, T)+\cdots \\
& V=1+\epsilon V^{\prime}(A, T)+\cdots \\
& X=A+\epsilon X^{\prime}(A, T)+\cdots
\end{aligned}
$$

Substituting into $\left(1^{\prime}\right),\left(2^{\prime}\right)$ and $\left(3^{\prime}\right)$, and ignoring powers of $\epsilon$ higher than the first, we obtain a linear system

$$
\begin{aligned}
V^{\prime} & =\frac{\partial X^{\prime}}{\partial A}, \\
\gamma \frac{\partial^{2} X^{\prime}}{\partial T^{2}} & =-\frac{\partial P^{\prime}}{\partial A}, \\
\gamma \frac{\partial V^{\prime}}{\partial T}+\frac{\partial P^{\prime}}{\partial T} & =I .
\end{aligned}
$$

For boundary and initial conditions, we have

$$
\begin{aligned}
& \text { at } T=0: P^{\prime}=V^{\prime}=X^{\prime}=\frac{\partial P^{\prime}}{\partial T}=\frac{\partial V^{\prime}}{\partial T}=\frac{\partial X^{\prime}}{\partial T}=0, \\
& \text { at } A=0: \frac{\partial X^{\prime}}{\partial T}=0, \\
& \text { at } A=1: P^{\prime}=0 .
\end{aligned}
$$

The independent variable $A$ may be used instead of the dependent variable $X$ in the end-point boundary conditions since $X=A$ to first order terms in the perturbation parameter $\epsilon$.

The linear system (i), (ii), (iii) may be most easily solved by introducing a generating function $W(A, T)$ such that

$$
\frac{\partial W}{\partial A}=2 \gamma\left(1-\lambda^{2}\right) \frac{\partial X^{\prime}}{\partial T} ; \quad \frac{\partial W}{\partial T}=2\left(1-\lambda^{2}\right) P^{\prime} .
$$

Then $(i i)$ is solved identically. Eliminating $V^{\prime}, P^{\prime}$ and $X^{\prime}$, we obtain a wave equation for $W$,

$$
\frac{\partial^{2} W}{\partial A^{2}}-\frac{\partial^{2} W}{\partial T^{2}}=2\left(1-\lambda^{2}\right) I
$$

The general solution of (6) will be of the form

$$
W=F(A+T)+G(A-T)+(A-\lambda T)^{2} I,
$$

where the functions $F$ and $G$ must be determined so as to satisfy the boundary conditions 


$$
\begin{array}{ll}
\text { at } A=0, & \frac{\partial W}{\partial A}=0, \\
\text { at } A=1, & \frac{\partial W}{\partial T}=0 .
\end{array}
$$

The initial conditions are that $W$ and its first partial derivatives be zero at $t=0$. We also require that $W$ and its first partial derivatives be continuous for $t>0$. In Fig. 2

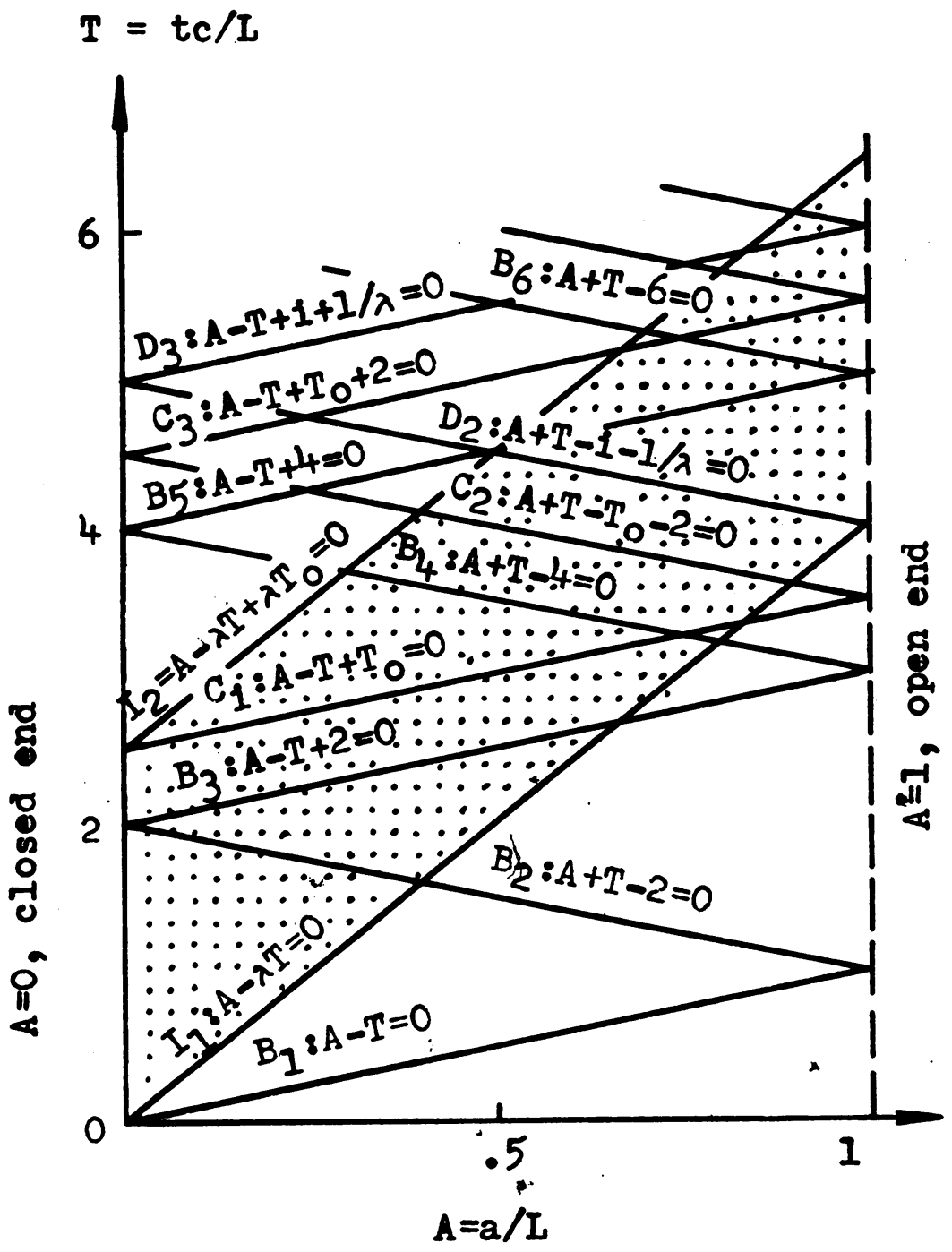

Fig. 2. Characteristic lines and burning zone in $(A, T)$ plane.

we indicate the region of interest in the $(A, T)$ plane, together with the important characteristic lines of the form $A \pm T=$ const.; the shaded region corresponds to the burning region. There are three independent sets of characteristic lines, labeled with 
the letters $B, C$ and $D$ respectively. It may be easily verified that the function $W$ which satisfies all the required conditions is given by

$$
\begin{aligned}
W=B_{1}^{*}(A-T)^{2} & +B_{2}^{*}(A+T-2)^{2}+B_{3}^{*}(A-T+2)^{2}+\cdots \\
& +I_{1}^{*}(A-\lambda T)^{2}+I_{2}^{*}\left(A-\lambda T+\lambda T_{0}\right)^{2} \\
& +C_{1}^{*}\left(A-T+T_{0}\right)^{2}+C_{2}^{*}\left(A+T-2-T_{0}\right)^{2}+\cdots \\
& +D_{2}^{*}(A+T-1-1 / \lambda)^{2}+\cdots
\end{aligned}
$$

where the quantities $B_{1}^{*}, C_{1}^{*}$, etc. are defined to be zero for all points $(A, T)$ below the corresponding characteristic lines and equal to certain constants above these lines (see Fig. 2). The constants are determined so that the boundary conditions are satisfied. For example: in the region in the $(A, T)$ plane near the closed end and just inside the burning region (see Fig. 2), $W$ is given by

$$
W=B_{1}(A-T)^{2}+I_{1}(A-\lambda T)^{2} .
$$

According to (7) and the definition of $I$, we have $I_{1}=1$. Then $B_{1}$ is to be determined so as to satisfy the boundary condition

$$
\frac{\partial W}{\partial A}=0 \quad \text { at } \quad A=0 .
$$

We have

$$
\left.\frac{\partial W}{\partial A}\right|_{A=0}=2 B_{1}(-T)+2(-\lambda T)=0
$$

and hence $B_{1}=-\lambda$. The other constants are determined in a similar fashion. The result is

$$
\begin{gathered}
-\lambda=B_{1}=-B_{2}=-B_{3}=B_{4}=B_{5}=-B_{6}=\cdots, \\
\lambda=C_{1}=-C_{2}=-C_{3}=\cdots \\
\lambda^{2} \quad=-D_{2}=-D_{3}=\cdots, \\
\quad I_{1}=1, \quad I_{2}=-1 .
\end{gathered}
$$

These constants completely specify the function $W$. The quantities $P, X$ and $M=$ $\partial X / \partial T=\partial x / c \partial t$ are then given in terms of $W$ by,

$$
\begin{aligned}
& P=1-\frac{\epsilon}{2\left(1-\lambda^{2}\right)} \frac{\partial W}{\partial T} \\
& X=A+\frac{\epsilon}{2 \gamma\left(1-\lambda^{2}\right)} \int_{0}^{T} \frac{\partial W}{\partial A} d T,
\end{aligned}
$$




$$
M=\frac{\epsilon}{2 \gamma\left(1-\lambda^{2}\right)} \frac{\partial W}{\partial A}
$$

4. Particular results. Special cases of (10), (11) and (12) which are of particular interest are $P(0, T), X(\lambda T, T)$ and $M(1, T)$ which give respectively the pressure at the closed end of the tube, the position of the flame front and the velocity of gas flow at the open end as functions of the time. These are given by

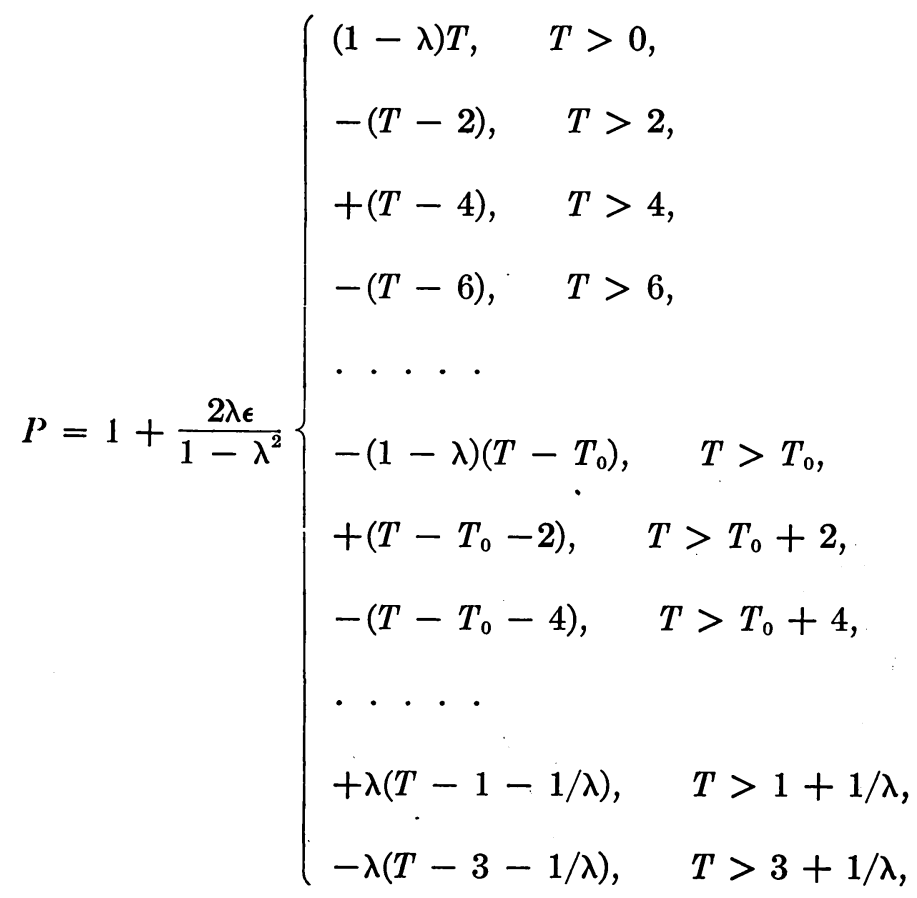

$$
\begin{aligned}
& X=\lambda T+\frac{\epsilon \lambda}{2 \gamma\left(1-\lambda^{2}\right)} \begin{cases}(1-\lambda)^{2} T^{2}, \quad T>0, \\
+(1+\lambda)^{2}(T-2 / 1+\lambda)^{2}, & T>2 / 1+\lambda, \\
-(1-\lambda)^{2}(T-2 / 1-\lambda)^{2}, & T>2 / 1-\lambda, \\
-(1+\lambda)^{2}(T-4 / 1+\lambda)^{2}, & T>4 / 1+\lambda, \\
-(1-\lambda)^{2}\left(T-T_{0} / 1-\lambda\right)^{2}, & T>T_{0} / 1-\lambda, \\
-(1+\lambda)^{2}\left(T-\frac{2+T_{0}}{1+\lambda}\right)^{2}, & T>\frac{2+T_{0}}{1+\lambda},\end{cases}
\end{aligned}
$$




$$
M=\frac{2 \lambda \epsilon}{\gamma\left(1-\lambda^{2}\right)}\left\{\begin{array}{l}
0, \quad T<1, \\
+(T-1), \quad T>1, \\
-(T-3), \quad T>3, \\
\cdot \cdot \cdot \quad T>5, \\
-\left(T-1-T_{0}\right), \quad T>1+T_{0} \\
+\left(T-3-T_{0}\right), \quad T>3+T_{0}, \\
\cdot \cdot \cdot
\end{array}\right.
$$

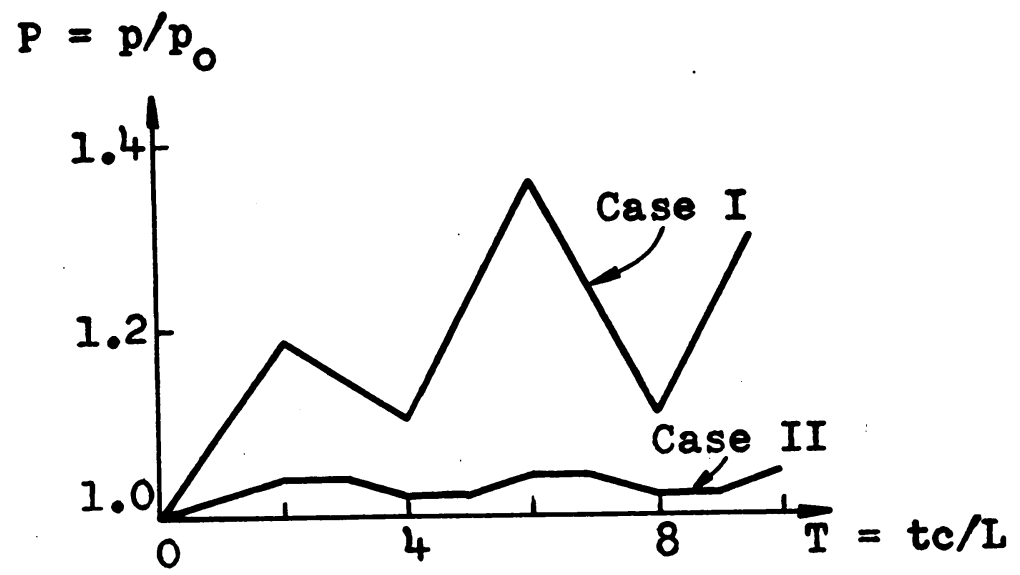

Fig. 3. Pressure at closed end of the tube for cases I and II.

In Figs. 3, 4 and 5 we give gràphs for $P, X$ and $M$ for two different sets of possibly typical numerical values for the parameters $\epsilon, \lambda$ and $T_{0}$, corresponding respectively to the fast relatively violent phenomena associated with turbulent initial conditions in the tube and to the more mild phenomena associated with static initial conditions in the tube. The two cases are given by, Case I: $\lambda=.3, \epsilon=.2, T_{0}=6$; Case II: $\lambda=.05, \epsilon=.2, T_{0}=3$. 
$T=c t / L$

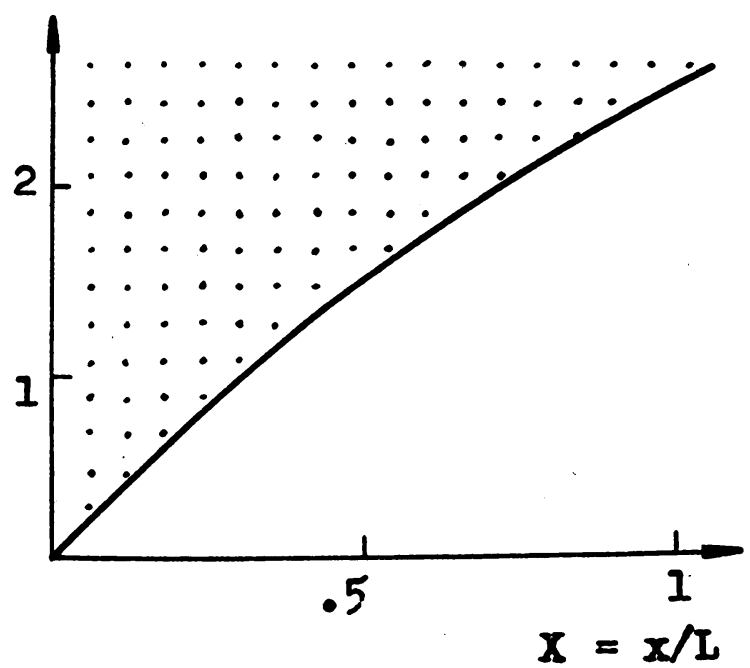

FIG. [4a. Position of the flame front with respect to the tube for Case I.

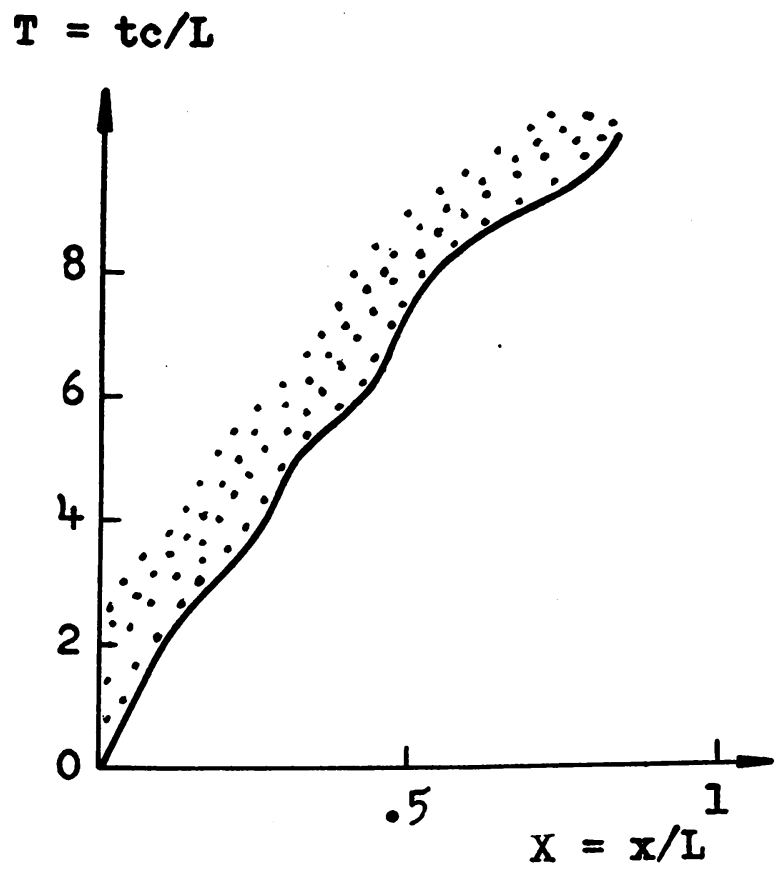

Fig. 4b. Position of the flame front with respect to the tube for Case II. 


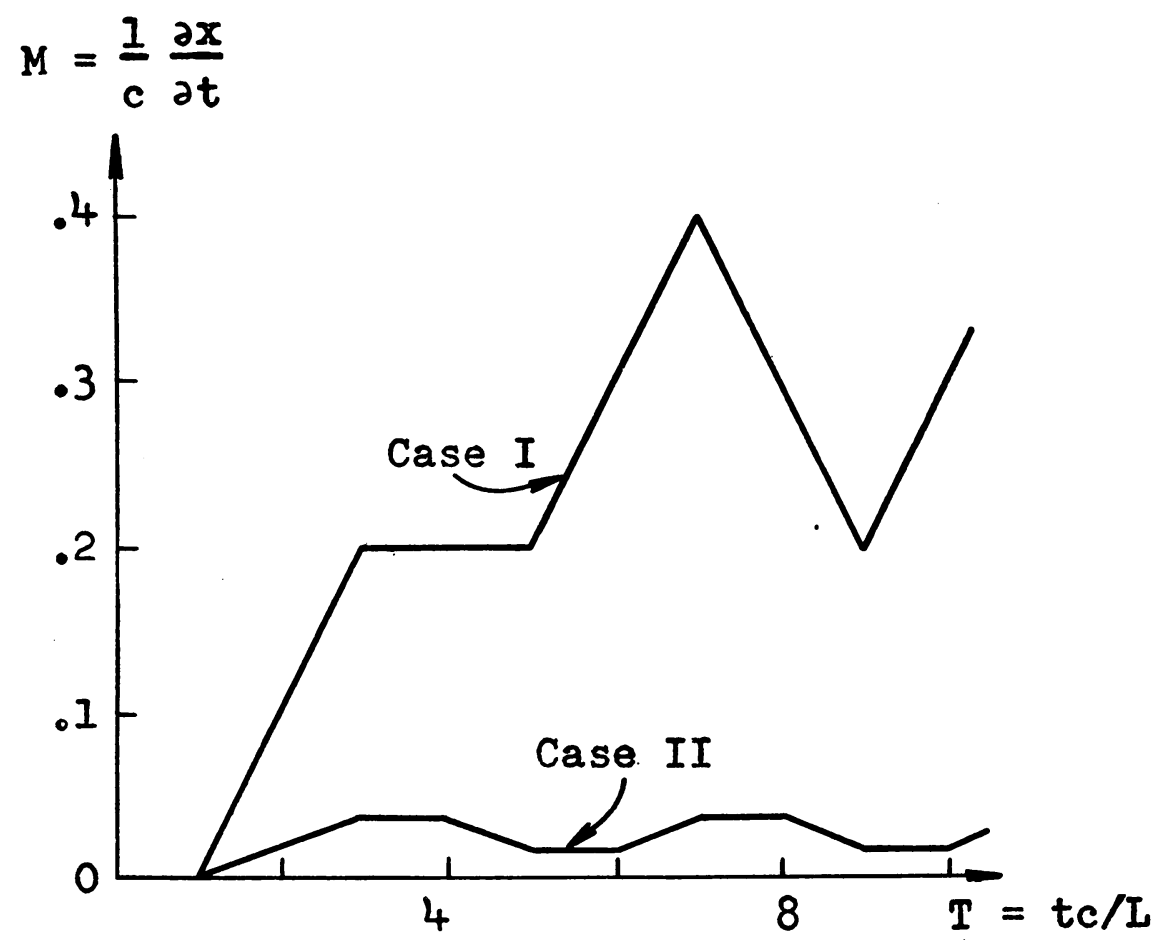

Fig. 5. Mach number $M=\partial x / c \partial t$ of the gas flow at the open end of the tube for Cases I and II. 